\title{
WAVE PROPAGATION IN A COAXIAL SYSTEM*
}

\author{
BY \\ V. M. PAPADOPOULOS
}

Brown University

\begin{abstract}
A solution is obtained for the problem of the propagation of electromagnetic waves in a semi-infinite flanged coaxial line with an infinite center conductor, in terms of an infinite set of coefficients which are determined by an infinite set of linear equations. The solution is discussed, in detail, in limiting cases which illustrate properties both of a thin vertical antenna on a plane perfectly conducting earth, and of a thick antenna fed by a low impedance line. Numerical results are given in these cases. The possibility of a solution for any excitation frequency is also discussed.

Introduction. The use of an antenna for the purpose of radiating electromagnetic energy must involve the generation of this energy and its passage to the antenna from the generator along a transmission line. There is, however, very little theoretical work published in which an antenna is considered with its means of excitation. It might appear to be an advantage to examine the radiating system in isolation, and then to regard it as an impedance lumped at the end of a transmission line: the magnitude of such an impedance depends, however, on the transmission line parameters. It is therefore of interest to find whether there is any range of parameters for which the line and the antenna are substantially independent. The work done on this subject by King and others [3] implies that as we reduce the spacing of the transmission line to zero we may extrapolate from a series of experimental measurements of physical quantities to the limiting case of zero spacing; this value may be identified with theoretical results obtained by assuming, in the isolated radiating system, a delta-function excitation (sometimes called a "slice" generator) at the driving point of the antenna. This statement needs qualification, since the question of the existence of the physical quantity in the limit of zero spacing was not considered by King. We find that this limit does not indeed exist. Accordingly, in this paper we are concerned with an idealization of the cylindrical antenna problem in which the complete transmission circuit may be examined mathematically. As an approach to the problem of the antenna of finite length, we shall consider a semi-infinite flanged coaxial line with its center conductor extended to an infinite length. A signal set up at one end of the line is partly reflected at the open end and partly radiated into the half-space outside the line. Since we are interested in antennae, we may describe the radiating system as an infinite vertical antenna of circular cross-section, standing on a horizontal, perfectly conducting ground of infinite extent, with the exciting signal fed in at the bottom.

We shall further simplify the system by considering the case in which the field is radially symmetric about the axis of the cylinder, with no magnetic field component parallel to this axis. The field components are related by Maxwell's equations; in this

*Received May 25, 1958: revised manuscript received January 12, 1959. The research described in this paper was partly supported by the Air Force Cambridge Research Center under Contract No. AF 19(604)-1391, and by the Office of Naval Research and the David W. Taylor Model Basin under Contract Nonr-562(24).
\end{abstract}


problem all the non-zero field components may be written down in terms of the transverse component of the magnetic field tangential to the surface of the cyclinder, and this component satisfies the scalar wave equation. The normal derivatives of the magnetic field vanish on all the perfectly conducing surfaces. The field components in the halfspace are related to the normal derivative of the magnetic field in the terminal plane of the coaxial line: this relationship may be found by a method in which cosine transforms are used. Since the field within the line may be expressed in terms of an infinite set of discrete modes (Marcuvitz [4]), it is possible by matching orthogonal components across the gap at the open end of the line to set up an infinite set of linear equations involving an infinite set of unknown coefficients. Each coefficient is related simply to the amplitude of the corresponding mode in the line; in turn, the set of coefficients determines all the field components completely and uniquely. To simplify the analysis, the free-space propagation constant is taken to have a small negative imaginary part which is later taken to be zero. The resulting solution is shown to satisfy the conditions of the problem.

The solution of the infinite set of equations is simplified when the spacing of the coaxial line is small compared to the wavelength of the exciting signal. This simplifying condition is valid either when the outer diameter of the line is a small fraction of the wavelength or when the spacing between the conductors in the line is a small fraction of the line diameter. With this simplification, we find that the field within the line is very nearly that in an open-circuited line, and this approximate field leads us to a good value both for the admittance of the radiating system and for the energy density at large distances from the antenna. The effect of the simplification is to give us results not only for the thick cylindrical antenna with a small line spacing but also for the thin antenna with a physically plausible method of excitation.

It should be emphasized that the results are appropriate to an idealized antenna of infinite length. The energy distribution may not be related to that of a finite antenna. On the other hand, the admittance of the infinite antenna is the limiting value of the admittance of long antennae.

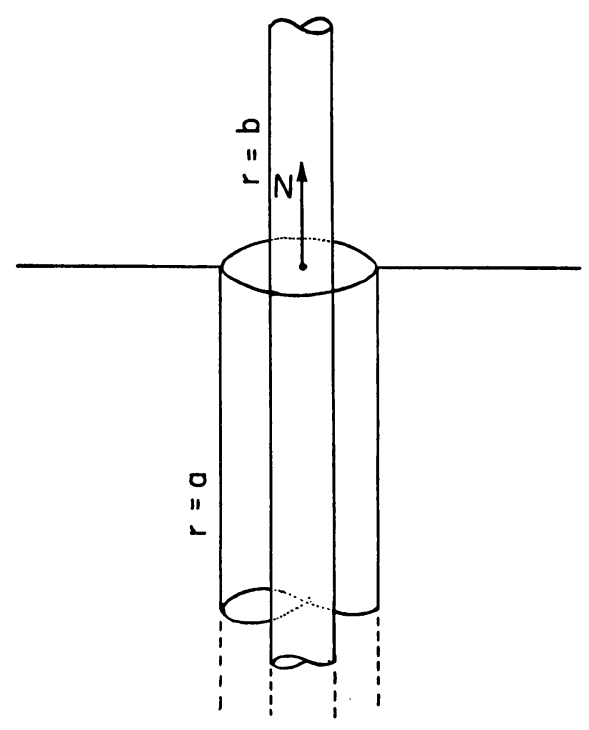

FIG. 1 
Results associated with a similar geometry without a flange have been given by Marcuvitz [4]. It is implied that these results were obtained by a method involving the use of the Wiener-Hopf technique. In the present problem a similar technique may well be practical, but the author has not yet made a comparison.

I. The geometry of the system to be considered is shown in Fig. 1. Cylindrical co-ordinates $(r, \theta, z)$ are used. The axis of an infinite, circular, conducting cylinder of radius $b>0$ is taken to be the $z$-axis. In the region $z<0$ this cylinder is surrounded by a semi-infinite coaxial conducting surface of radius $a(a>b)$, and this surface, $r=a$, $z<0$, is terminated in the plane $z=0$ by a perfectly conducting plane in the region $r>a, z=0$. A time dependence exp $(i \omega t)$ is assumed throughout, and rationalized m.k.s. units are used.

We are to consider the electromagnetic field in the region bounded by the conducting surfaces corresponding to a combination of axially symmetric transverse magnetic modes in the line region $a>r>b, z<0$. This type of field is independent of the co-ordinate $\theta$, and it has the magnetic field components $B_{r}$ and $B_{z}$ both zero. From Maxwell's equations, it follows that the electric field components are related to the magnetic field component $B_{\theta}$ by the equations,

$$
\begin{aligned}
\frac{i k^{2}}{\omega} E_{z} & =\frac{1}{r} \frac{\partial}{\partial r}\left(r B_{\theta}\right), \\
\frac{i k^{2}}{\omega} E_{r} & =-\frac{\partial B_{\theta}}{\partial z}, \\
E_{\theta} & =0
\end{aligned}
$$

where $k$ is taken with a small negative imaginary part $\left(k=k_{r}-i k_{i}, k_{i}, k_{r}>0\right)$, and $B_{\theta}$ must satisfy the equation,

$$
\left(\frac{\partial}{\partial r^{2}}+\frac{1}{r} \frac{\partial}{\partial r}+\frac{\partial^{2}}{\partial z^{2}}+k^{2}-\frac{1}{r^{2}}\right) B_{\theta}=0 .
$$

Since the tangential component of the electric field vanishes at a perfectly conducting surface, $B_{\theta}$ must satisfy the boundary conditions

$$
\begin{aligned}
& \frac{\partial}{\partial r}\left(r B_{\theta}\right)=0 \quad \text { on } \quad r=b, \\
& \frac{\partial}{\partial r}\left(r B_{\theta}\right)=0 \quad \text { on } \quad r=a, \quad z<0 \\
& \frac{\partial}{\partial z}\left(B_{\theta}\right)=0 \quad \text { on } \quad z=0, \quad r>a .
\end{aligned}
$$

We shall assume the total field to be made up of an incident wave in the dominant mode within the coaxial line, with magnetic component $B_{\theta_{0}}(r, z)$ and a scattered field with magnetic components $B_{\theta_{+}}(r, z)$ for $z>0$, and $B_{\theta_{-}}(r, z)$ for $z<0$. $B_{\theta_{0}}$ is defined by the equation,

$$
B_{\theta_{0}}=\exp (-i k z) / r \text { for } a>r>b, \quad z<0 .
$$

With $k_{i}>0$, the radiation condition at infinity is satisfied if we take $B_{\theta_{-}}$to be of backward-travelling wave form within the line, and $B_{\theta_{+}}$to have a wave form travelling away 
from the open end of the line. Thus, in the half-space $z>0$ the behavior of $B_{\theta_{+}}$is given by the relation

$$
R B_{\theta_{+}} \sim f(\varphi) \exp (-i k R) \quad \text { as } \quad R \rightarrow \infty, \quad 0<\varphi<\pi / 2,
$$

where $R=\left(r^{2}+z^{2}\right)^{1 / 2}, \varphi=\tan ^{-1}(r / z)$, and $f(\varphi)$ is a bounded function independent of $R$. At the edge $r=a, z=0$, the condition on the total field which ensures the integrability of the edge current and hence the uniqueness of the solution $[2,5]$ is that $B_{\theta}=$ constant $-0\left(\sigma^{2 / 3}\right)$, and $\partial B_{\theta} / \partial z=0\left(\sigma^{-1 / 2}\right)$ as the distance $\sigma$ of the point of observation to the edge tends to zero. The problem is now completely and uniquely specified.

II. To find the field in the half-space $z>0$, we define a Fourier cosine transform for all $r>b$ by the equation,

$$
F(p, r)=\int_{0}^{\infty} B_{\theta_{+}}(r, z) \cos p z d z .
$$

The absolute value of this integral for large values of $z$ is found from Eq. 7 to contain the factor exp $-k_{i}\left(r^{2}+z^{2}\right)^{1 / 2}$, and this factor ensures the absolute convergence of the integral. The inverse of Eq. 8 is given by

$$
B_{\theta_{+}}(r, z)=2 \int_{0}^{\infty} F(p, r) \cos p z d p / \pi
$$

for all $r>b$.

If we multiply Eq. 2 by $\cos p z$ and integrate with respect to $z$ in the range $z>0$, we find that

$$
\left(\frac{\partial^{2}}{\partial r^{2}}+\frac{1}{r} \frac{\partial}{\partial r}+K^{2}-\frac{1}{r^{2}}\right) F(p, r)=\left(\frac{\partial B}{\partial z}\right)_{z=0}
$$

so that for $r>a$, from Eq. $5, F(p, r)$ satisfies the equation

$$
\left(\frac{\partial^{2}}{\partial r^{2}}+\frac{1}{r} \frac{\partial}{\partial r}+K^{2}-\frac{1}{r^{2}}\right) F(p, r)=0,
$$

and for $a>r>b$, writing $L(r)$ for $\left(\partial B_{\theta} / \partial z\right)_{z=0}, F(p, r)$ satisfies the equation

$$
\left(\frac{\partial^{2}}{\partial r^{2}}+\frac{1}{r} \frac{\partial}{\partial r}+K^{2}-\frac{1}{r^{2}}\right) F(p, r)=L(r) .
$$

Here $K=\left(k^{2}-p^{2}\right)^{1 / 2}$; that branch of the square root is taken for which when $k_{i}=0$, $K$ is real and positive when $p$ is real and $|p|<k$, and for which $i K$ is real and positive when $p$ is real and $|p|>k$.

From Eq. $3, \partial[r F(p, r)] / \partial r$ must vanish at $r=b$. On the surface $r=a, B_{\theta}$ and $\partial B_{\theta} / \partial z$ are functions of integrable square for all $z>0$ : the continuity of the field components $B_{\theta}$ and $E_{z}$ across this surface therefore ensures the continuity of the functions $F(p, r)$ and $\partial[r F(p, r)] / \partial r$ at $r=a$. From Eqs. 7 and 8 it follows that as $r \rightarrow \infty$

$$
F(p, r) \sim \int_{0}^{\infty} f(\varphi) \exp (-i k R) \cos p z d z / R .
$$

For large values of $r$ this integral may be evaluated by the method of steepest descents: the calculation shows that

$$
F(p, r) \sim \psi(p) \exp (-i k r) / r^{1 / 2},
$$

where $\psi(p)$ is a function of $p$ only. 
The solution of Eq. 10 which shows this behavior for large values of $r$ is a multiple of $H_{1}(K r) ; H_{1}(K r)$ is that solution of Bessel's equation for which as $r \rightarrow \infty$ for real or imaginary values of $K$

$$
(K r)^{1 / 2} H_{1}(K r) \sim \exp [-i(K r-3 \pi / 4)][2 / \pi]^{1 / 2} .
$$

When $K$ is real this is the Hankel function of $H_{1}^{(2)}(K r)$. Similarly, $H_{0}(K r)$ is a zero order Bessel function which for real values of $K$ is to be identified with the Hankel function $H_{0}^{(2)}(K r)$.

The solutions of Eqs. 10 and 11 which satisfy the required boundary and continuity conditions for $F(p, r)$ and $\partial[r F(p, r)] / \partial r$ are, for $r>a$.

$$
F(p, r)=\pi H_{1}(K r) \int_{b}^{a} \rho L(\rho)\left[J_{1}(K \rho) Y_{0}(K b)-Y_{1}(K \rho) J_{0}(K b)\right] d \rho / 2 H_{0}(K b),
$$

and for $b<r<a$,

$$
\begin{aligned}
& 2 F(p, r)\left[J_{0}(K a) Y_{0}(K b)-Y_{0}(K a) J_{0}(K b)\right] / \pi=\left[J_{1}(K r) Y_{0}(K b)-Y_{0}(K a) J_{0}(K b)\right] \\
& \cdot \int_{b}^{a} \rho L(\rho) H_{0}(K a)\left[J_{1}(K) Y_{0}(K b)-Y_{1}(K \rho) J_{0}(K b)\right] d \rho / H_{0}(K b) \\
&- {\left[J_{1}(K r) Y_{0}(K b)-Y_{1}(K r) J_{0}(K b)\right] } \\
& \cdot \int_{r}^{a} \rho L(\rho)\left[J_{1}(K \rho) Y_{0}(K a)-Y_{1}(K \rho) J_{0}(K a)\right] d \rho \\
&- {\left[J_{1}(K r) Y_{0}(K a)-Y_{1}(K r) J_{0}(K a)\right] } \\
& \cdot \int_{b}^{r} \rho L(\rho)\left[J_{1}(K \rho) Y_{0}(K b)-Y_{1}(K \rho) J_{0}(K b)\right] d \rho .
\end{aligned}
$$

These equations determine the cosine transforms of the magnetic field in the half-space in terms of the function $L(r)$ which is proportional to the radial electric field in the gap $z=0, a>r>b$.

From Eqs. 9 and 13 we may write down the total magnetic field in the gap. This is

$$
\begin{aligned}
& B_{\theta_{+}}(r, \theta)=\int_{0}^{\infty} d \rho\left\{\frac{J_{1}(K r) Y_{0}(K b)-Y_{1}(K r) J_{0}(K b)}{J_{0}(K a) Y_{0}(K b)-Y_{0}(K a) J_{0}(K b)}\right\} \frac{H_{0}(K a)}{H_{0}(K b)} \\
& \cdot \int_{b}^{a} \rho L(\rho)\left[J_{1}(K \rho) Y_{0}(K b)-Y_{1}(K \rho) J_{0}(K b)\right] d \rho-\frac{J_{1}(K r) Y_{0}(K a)-Y_{1}(K r) J_{0}(K a)}{J_{0}(K a) Y_{0}(K b)-Y_{0}(K a) J_{0}(K b)} \\
& \cdot \int_{b}^{r} \rho L(\rho)\left[J_{1}(K \rho) Y_{0}(K b)-Y_{1}(K \rho) J_{0}(K b)\right] d \rho-\frac{J_{1}(K r) Y_{0}(K b)-Y_{1}(K r) J_{0}(K b)}{J_{0}(K a) Y_{0}(K b)-Y_{0}(K a) J_{0}(K b)} \\
& \cdot \int_{r}^{a} \rho L(\rho)\left[J_{1}(K \rho) Y_{0}(K a)-Y_{1}(K \rho) J_{0}(K a)\right] d \rho .
\end{aligned}
$$

III. In the region $z<0, a>r>b$, the field can be described in terms of a set of axially symmetric transverse magnetic modes. These modes are associated with the set of functions $r^{1 / 2} \varphi_{n}(r)$, orthogonal in $a>r>b$, where

and

$$
\varphi_{0}(r)=1 / r
$$

$$
\varphi_{n}(r)=J_{1}\left(K_{n} r\right) Y_{0}\left(K_{n} a\right)-Y_{1}\left(K_{n} r\right) J_{0}\left(K_{n} a\right),
$$


for integer values of $n \geq 1$. When $n \geq 1, K=K_{n}$ is the $n$th positive zero in order of magnitude of the function $J_{0}(K a) Y_{0}(K b)-Y_{0}(K a) J_{0}(K b)$, and $K_{0}=0$. For all $n>1$, $(a-b) K_{n} \sim n \pi$ [1]. If we put $p_{n}$ to be that value of $p$ which corresponds to the value $K=K_{n}$, then the magnetic field component of any axially symmetric $T M$ field within the line is a linear combination of modes of the form $\varphi_{n}(r) \exp \left( \pm i p_{n} z\right)$.

We have assumed that an incident dominant mode $B_{\theta_{0}}$ of unit amplitude travels towards the open end of the line. If $R_{n}$ is the reflexion coefficient associated with the $n$th mode reflected at the plane $z=0$, for an incident dominant mode of unit amplitude, then we may write the reflected field in the form

so that

$$
B_{\theta_{-}}=\sum_{n=0}^{\infty} R_{n} \varphi_{n}(r) \exp \left(i p_{n} z\right),
$$

$$
\delta B_{\theta_{-}} / \delta z=\sum_{n=0}^{\infty} i p_{r} R_{n} \varphi_{n}(r) \exp \left(i p_{n} z\right) .
$$

By matching both the total magnetic field and its normal derivative across the open end of the line we find that

and

$$
B_{\theta_{+}}(r, 0)=\sum_{n=0}^{\infty}\left(R_{n}+\delta_{0 n}\right) \varphi_{n}(r),
$$

$$
L(r)=\sum_{n=0}^{\infty} i p_{n}\left(R_{n}-\delta_{0 n}\right) \varphi_{n}(r),
$$

where $\delta_{m n}=0$ for $m \neq n$, and $\delta_{m m}=1$ for all integer values of $m$. Both the functions $r^{1 / 2} L(r)$ and $r^{1 / 2} B_{\theta_{+}}(r, 0)$ are functions of integrable square in $a>r>b$, so that they may be expanded in orthogonal series of the functions $\varphi_{n}(r)$.

Thus, if in $a>r>b$

then

$$
L(r)=\sum_{n=0}^{\infty} a_{n} \varphi_{n}(r),
$$

where

$$
a_{n} N_{n}=\int_{b}^{a} r L(r) \varphi_{n}(r) d r
$$

$$
\begin{gathered}
N_{n}=\int_{b}^{a} r\left[\varphi_{n}(r)\right]^{2} d r . \\
\left\{\begin{array}{l}
N_{0}=\ln (a / b) \\
N_{n}=2\left[1-\left[J_{0}\left(K_{n} a\right) / J_{0}\left(K_{n} b\right)\right]^{2}\right] /\left(\pi K_{n}\right)^{2}
\end{array}\right\} .
\end{gathered}
$$

We can see therefore from Eqs. 18 and 19 that

and from Eq. 17, that

$$
a_{n}=i p_{n}\left(R_{n}-\delta_{0 n}\right),
$$

$$
\int_{b}^{a} r B_{\theta+}(r, 0) \varphi_{n}(r) d r=N_{n}\left(R_{n}+\delta_{0 n}\right) .
$$

Since we are able to write $B_{\theta_{+}}(r, 0)$ in terms of the coefficients $a_{n}$, Eq. 21 may be manipulated to represent an infinite set of equations linear in the $a_{n}$ whose solution will determine the field throughout the system. 
Thus, from Eqs. 14 and 19, assuming that the required changes in the orders of integration and summation are permissible, it follows that

where

$$
k \int_{b}^{a} r B_{\theta+}(r, 0) \varphi_{m}(r) d r=\sum_{n=0}^{\infty} C_{m n} a_{n},
$$

$$
\begin{aligned}
C_{m n}=k \int_{0}^{\infty} d p\left\{K^{2} a^{2} \frac{H_{0}(K a) \varphi_{m}(a) \varphi_{n}(a)}{H_{0}(K b)\left(K^{2}-K_{n}^{2}\right)\left(K^{2}-K_{m}^{2}\right)}\right. \\
\left.\cdot\left[J_{0}(K a) Y_{0}(K b)-Y_{0}(K a) J_{0}(K b)\right]+\frac{2 N_{n} \delta_{n m}}{\pi K^{2}-\pi K_{n}^{2}}\right\} .
\end{aligned}
$$

It follows from Eqs. 21 and 22 that the infinite set of equations relating the coefficients $a_{n}$ is

for all integer $n \geq 0$.

$$
2 \delta_{0 m} N_{m}+a_{n} N_{m} /\left(i p_{m}\right)=\sum_{n=0}^{\infty} C_{m n} a_{n} / k,
$$

IV. In the complex $p$-plane the integrand defined in Eq. 23 has for singularities only branch points at $p= \pm k_{0}$. It is clear that the path of integration for the integral $C_{m n}$ must be deformed into an equivalent contour: this is necessary in order to avoid passing through the branch point $p= \pm k$ in the limiting case when $k_{i}=0$. Thus, in the neighborhood of this branch point we can deform the path of integration into a semi-circular arc of small radius $\delta$ in the upper half $p$-plane. To examine the contribution to the integral $C_{m n}$ from this arc we put $p=k+\delta \exp i \varphi, 0 \leq \varphi \leq \pi$, where we can now take $k_{i}$ to be zero. Since as $\delta \rightarrow 0$ the corresponding form for $K$ is

$$
K=(2 k \delta)^{1 / 2} \exp i(\theta-\pi) / 2[1+0(\delta)],
$$

we can show that

$$
\pi\left[J_{0}(K a) Y_{0}(K b)-Y_{0}(K a) J_{0}(K b)\right] / 2=\ln b / a+0[\delta(a-b)] ;
$$

it follows that for $m=n=0$, when $N_{0}=\ln a / b$, the absolute value of the integrand for small values of $\delta$ is $0(1)$, and the contribution to the integral $C_{00}$ is $0(\delta)$. For $m$ or $n$ not zero the contribution may be shown to be even smaller in magnitude. We may therefore write $C_{m n}$ in the form of a line integral, this being the limiting case for $\delta=0$. The question of the convergence of this integral is considered in the Appendix. It is shown there that $C_{m n}$ is uniformly convergent with respect to the parameters $a$ and $b$ in the range $a>b>0$. Since we have defined the branch of $k$ so that for $p$ real, $0<p<k, k$ is real, and for $p>k, k=-i \mu$ where $\mu$ is real, it follows that

$$
\begin{aligned}
\frac{C_{m n}}{k}=\int_{0}^{k} d p\left\{K^{2} a^{2} \frac{H_{0}(K a) \varphi_{m}(a) \varphi_{n}(a)}{H_{0}(K b)\left[K^{2}-K_{m}^{2}\right]\left(K^{2}-K_{n}\right)^{2}}\right. \\
\left.\cdot\left[J_{0}(K a) Y_{0}(K b)-Y_{0}(K a) J_{0}(K b)\right]+\frac{2}{\pi} \frac{N n \delta n m}{K^{2}-K_{m}^{2}}\right\} \\
+\frac{2}{\pi} \int_{0}^{\infty} d p\left\{\mu^{2} a^{2} \frac{K_{0}(\mu a)}{K_{0}(\mu b)} \frac{\varphi_{m}(a) \varphi_{n}(a)}{\left(\mu^{2}+K_{m}^{2}\right)\left(\mu^{2}+K_{n}^{2}\right)}\right. \\
\left.\cdot\left[I_{0}(\mu a) K_{0}(\mu b)-I_{0}(\mu b) K_{0}(\mu a)\right]-\frac{N n \varphi m n}{K_{m}^{2}+\mu^{2}}\right\},
\end{aligned}
$$


where $K=\left(k^{2}-p^{2}\right)^{1 / 2}, \mu=\left(p^{2}-k^{2}\right)^{1 / 2}$, and $K_{0}(z), I_{0}(z)$ are modified Bessel functions [6].

V. The first quantity of physical interest to be considered is the energy density at large distances from the antenna. From Eqs. 9 and 12 it may be seen that for $r>a$,

$$
B_{\theta_{+}}(r, z)=\int_{0}^{\infty} \cos p z H_{1}(k r) \int_{b}^{a} L(\rho)\left[J_{1}(K r) Y_{0}(K b)-Y_{1}(K r) J_{0}(K b)\right] d \rho d p / H_{0}(K b),
$$

so that as $r \rightarrow \infty$, since the integrand is an even function of $p$, we have that

$$
\begin{aligned}
& B_{\theta_{+}}(r, z) \sim \text { const. } \int_{-\infty}^{\infty} \frac{d p \exp (i p z-i K r)}{(K r)^{1 / 2} H_{0}(K b)} \\
& \qquad \cdot \int_{b}^{a} \rho L(\rho)\left[J_{1}(K r) Y_{0}(K b)-Y_{1}(K r) J_{0}(K b)\right] d \rho .
\end{aligned}
$$

This integral may be evaluated by the method of steepest descents. By putting $R=\left(r^{2}+z^{2}\right)^{1 / 2}, \varphi=\tan ^{-1} r / z$ with $0<\varphi<\pi / 2$, we find that the saddle point is at $p=-k \cos \varphi$, corresponding to the value $k=k \sin \varphi$. The path of integration is deformed, without changing the value of the integral, into the steepest descent path, which at the saddle-point makes an angle of $\pi / 4$ with the line joining the branch points. The value of $B_{\theta_{+}}$for large values of $R$ can now be given. This is

$$
\begin{aligned}
B_{\theta+} \sim \text { const. } \frac{\exp (-i k R)}{k R H_{0}^{(2)}(k b \sin \varphi)} \int_{b}^{a} \rho L(\rho)\left[J_{1}(k \rho \sin \varphi) Y_{0}(k b \sin \varphi)\right. \\
\left.-Y_{1}(k \rho \sin \varphi) J_{0}(k b \sin \varphi)\right] d \rho,
\end{aligned}
$$

and from Eq. 19 after performing the integration, we find that

$$
\begin{aligned}
B_{\theta_{+}} \sim \frac{\text { const. } \sin \varphi \exp (-i k R)}{k R H_{0}^{(2)}(k b \sin \varphi)} & {\left[J_{0}(k a \sin \varphi) Y_{0}(k b \sin \varphi)\right.} \\
& \left.-Y_{0}(k b \sin \varphi) J_{0}(k a \sin \varphi)\right] \sum_{0}^{\infty} \frac{a_{n} a \varphi_{n}(a)}{k^{2} \sin ^{2} \varphi-K_{n}^{2}} .
\end{aligned}
$$

The energy density at large distances from the open end of the line can now be found from the complex Poynting vector, and it follows that the power flow $P$ in the radial direction at large distances is given by

$$
\begin{aligned}
P=\text { const. } \frac{\sin ^{2} \varphi\left\{J_{0}(K a \sin \varphi) Y_{0}(K b \sin \varphi)-Y_{0}(K b \sin \varphi) J_{0}(K a \sin \varphi)\right\}^{2}}{R^{2}\left\{\left[J_{0}(K b \sin \varphi)\right]^{2}+\left[Y_{0}(K b \sin \varphi)\right]^{2}\right\}} \\
\cdot\left\{\sum_{0}^{\infty} \frac{a_{n} a \varphi_{n}(a)}{k^{2} \sin ^{2} \varphi-K_{n}^{2}}\right\}^{2} .
\end{aligned}
$$

The second quantity which is of interest to us is the admittance of the antenna, regarded as a termination of the transmission line. If we go far enough along the line for higher order modes to be practically damped and then carry out standing wave measurements, we may determine the dominant mode reflexion coefficient $R_{0}$. The terminal admittance $Y_{1}$ associated with this coefficient $R_{0}$ is given by the equation

$$
Y_{1}=Y_{0}\left(R_{0}+1\right)\left(R_{0}-1\right)^{-1},
$$

where the characteristic admittance of the line $Y_{0}=2 \pi \omega \epsilon_{0} / k \ln (a / b)$. From Eqs. 20 
and 24 , it follows that

$$
Y_{1}=i Y_{0} \cdot \sum_{n=0}^{\infty} C_{0 n} a_{n} / a_{0} N_{0} .
$$

In an isolated radiating system the only way in which a driving-point admittance may be defined is by the ratio of current to voltage at this point. Thus, in this system if we define an admittance $Y_{2}$ to be the ratio of the current at the foot of the antenna to the voltage applied between the ground and the foot, then it follows, from expressions which can easily be found for the current and the voltage, that

$$
Y_{2}=i Y_{0} b \sum_{m=0}^{\infty} \sum_{n=0}^{\infty} C_{n m} a_{m} \varphi_{n}(b) / a_{0} N_{n} .
$$

The first term of this series is the admittance $Y_{1}$.

VI. The exact solution to this problem for general values of the quantities $k, a$, and $b$ involves the solution of an infinite set of linear equations whose coefficients are infinite integrals. Even with the help of modern computing machinery the work would be exceedingly difficult. We shall first consider, however, the solution for small values of the parameter $\epsilon=k(a-b)$. If we put $\sigma=k a$, and $\rho=b / a$, then it is clear that $\epsilon$ may be small if either $\sigma \rightarrow 0, \rho \neq 1$ or if $\sigma \neq 0, \rho \rightarrow 1:$ the first case corresponds to the physical problem of the antenna which is thin in comparison with the excitation wavelength, and the second to the problem of the thick antenna with a low impedance line feed. We will exclude, however, the case for which $\rho \rightarrow 1$ and $\sigma \rightarrow 0$, this being the case of the thin antenna with a low impedance feed.

For the limiting cases $\sigma \rightarrow 0, \rho \neq 1, \sigma \neq 0, \rho \rightarrow 1$ the magnitudes of the functions which appear in the infinite set of equations are derived in the Appendix. To solve the problem when $\epsilon \rightarrow 0$, we first assume that in the equations $24, a_{n}=0$ for $n \geq 1$. Then from the first equation of the set, it follows that

$$
\begin{aligned}
a_{0}=-2 i k, & \text { as } \rho \rightarrow 1, & \sigma \neq 0, \\
& \text { or } \sigma \rightarrow 0, & \rho \neq 1 .
\end{aligned}
$$

If we take this first approximation, we find from the $(n+1)$ th equation that

$$
\begin{aligned}
a_{r} & =\frac{i p_{r} C_{r 0}}{N} a_{0}, \\
& =0(1) \quad \text { as } \rho \rightarrow 1, \quad \sigma \neq 0, \quad \text { or } \quad \sigma \rightarrow 0, \quad \rho \neq 1 .
\end{aligned}
$$

Using these results for the orders of magnitude of the $a_{r}$ in the complete set of equations, we find that as $\epsilon \rightarrow 0$

$$
\begin{aligned}
& a_{0}=-2 i k[1+0(\epsilon)], \\
& a_{r}=0(1), \quad r \geq 1 .
\end{aligned}
$$

We now find in the expressions for the physically interesting quantities that $a_{0}=-2 i k$ is the only coefficient to make a significant contribution. This value for $a_{0}$ is that which would arise in analysis of an open-circuited line ignoring end effects.

From Eq. 19, the unknown function may now be given in the form

$$
L(r)=-2 i k / r .
$$




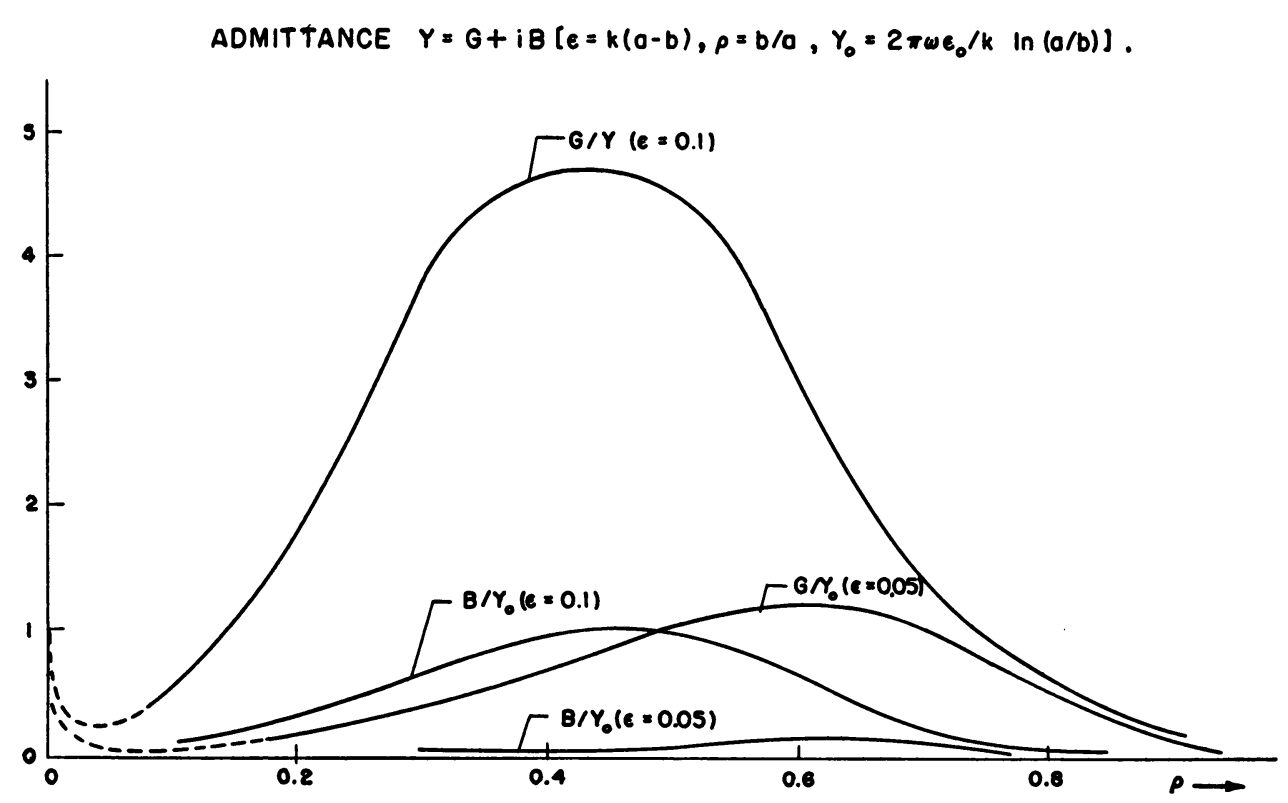

FIG. 2

The corresponding admittance of the radiating system, from either definition in Eq. 30 or 31 takes the form

$$
Y=i k Y_{0} C_{00}[1+0(\epsilon)] / N_{0} \text {. }
$$

The value of $Y$ in the limit as $\sigma \rightarrow 0, \rho \neq 1$ is $0(\epsilon)$. The limit as $\rho \rightarrow 1, \sigma \neq 0$ does not exist because the second derivative of $C_{00}$ with respect to $\rho$ does not exist. In this case the strongest statement to be made is that the normalized admittance $Y / Y_{0}$ is zero.

Numerical values of the admittance for small values of $\epsilon$ are given in the accompanying graph (Fig. 2). The normalized admittance approaches unity as $\rho \rightarrow 0$ for fixed small values of $\sigma$. The open-circuit approximation used to calculate results is not therefore applicable for small values of $\rho$. From Equation 29, when $\epsilon \rightarrow 0$ we find that

$$
P=\frac{2 \omega}{\mu k^{2} r^{2}} \frac{\left[J_{0}(\sigma \sin \varphi) Y_{0}(\sigma \rho \sin \varphi)-Y_{0}(\sigma \sin \varphi) J_{0}(\sigma \rho \sin \varphi)\right]^{2}}{\left[J_{0}(\sigma \rho \sin \varphi)\right]^{2}+\left[Y_{0}(\sigma \rho \sin \varphi)\right]^{2}}[1+0(\epsilon)] .
$$

For $r>a$ at large distances from the end of the line, where $\tan \varphi=r / z$ this expression may be simplified in certain cases.

In the case when $\sigma \rightarrow 0 \rho \neq 1$

$$
P \approx 2 \omega[\ln \rho]^{2} / \mu k^{2} r^{2}\left\{[\ln (\sigma \rho \sin \varphi) / 2]^{2}+\pi^{2} / 4\right\} .
$$

In the case when $\rho \rightarrow 1, \sigma \neq 0$ is more interesting. Thus

$$
P \approx 8 \omega(1-\rho)^{2} / \mu k^{2} r^{2} \pi^{2}\left\{\left[J_{0}(\sigma \rho \sin \varphi)\right]^{2}+\left[Y_{0}(\sigma \rho \sin \varphi)\right]^{2}\right\},
$$

and when $\sin \varphi \approx r / z$ for small enough values, $r>a$,

$$
P \approx 8 \omega(1-\rho)^{2} / \mu k^{2} r^{2} \pi^{2}\left\{4[\ln (\sigma r / 2 z) / \pi]^{2}+1\right\} .
$$

This expression shows that we may expect a behavior for $P$ like $[1 / r \ln r]^{2}$ for short wave- 
lengths when $\sigma=k a=0(z)$ for large values of $z$. More generally, if, when $\rho \rightarrow 1$ and sin $\varphi$ is not small, we take values of $\sigma$ which are large, but not so large that the relation $\sigma(1-\rho)=\epsilon \rightarrow 0$ is not satisfied, we find that

$$
P \approx 4 \omega \epsilon(1-\rho) \sin \varphi / \mu \pi k^{2} r^{2} .
$$

VII. In the general case, for $\epsilon>0$, we shall prove two results. The first is

Theorem 1. The infinite set of equations given in Eq. 24 has for a solution at most one set of values $a_{n}$ if $\epsilon=k a(a b)>0$.

It will be recalled that $a_{n}$ is a coefficient in the orthogonal expansion in $a>r>b$ of the function $r^{1 / 2} L(r)$.

Suppose that we have fixed $r \pi<\epsilon \leq \pi(r+1)$ so that $p_{m}(0 \leq m \leq r)$ is real and $i p_{m}=q_{m}(m \geq r+1)$ is also real. The homogeneous set of equations corresponding to Eq. 24 is

$$
k N_{m} a_{m} / i p_{m}=\sum_{n=0}^{\infty} C_{n m} a_{m}, \text { for } m \geq 0 .
$$

Putting $\alpha_{m}+i \beta_{m}=a_{m}, C_{m n}=K_{m n}+i L_{m n}$ we find that

$$
\begin{aligned}
\sum_{n=0}^{\infty}\left(K_{m n} \alpha_{n}-L_{m n} \beta_{n}\right) & =k N_{m} \beta_{m} / p_{m}, \quad \text { for } m \leq r, \\
& =k N_{m} \alpha_{m} / q_{m}, \text { for } m \geq r+1 . \\
\sum_{n=0}^{\infty}\left(K_{n m} \beta_{n}+L_{m n} \alpha_{n}\right)=-k N_{m} \alpha_{m} / p_{m}, & \text { for } m \leq r, \\
=k N_{m} \beta_{m} / q_{m}, & \text { for } m \geq r+1 .
\end{aligned}
$$

It follows that

$$
\begin{aligned}
-\beta_{m} \sum_{n=0}^{\infty} L_{m n} \beta_{n}-\alpha_{m} \sum_{n=0}^{\infty} L_{m n} \alpha_{n} & =0, \text { for } m \geq r+1, \\
& =k N_{m}\left(\alpha_{m}^{2}+\beta_{m}^{2}\right) / p_{m}, \text { for } m \leq r,
\end{aligned}
$$

so that

$$
k \sum_{0} N_{m}\left(\alpha_{m}^{2}+\beta_{m}^{2}\right) / p_{m}=-\sum_{m=0}^{\infty} \sum_{n=0}^{\infty}\left(\beta_{m} L_{m n} \beta_{n}+\alpha_{m} L_{m n} \alpha_{n}\right) .
$$

Now

$$
L_{m n}=2 k \int_{0}^{k} \frac{\varphi_{m}(a) \varphi_{n}(a) K^{2} a^{2}\left[J_{0}(K a) Y_{0}(K b)-Y_{0}(K a) J_{0}(K b)\right]^{2}}{\left(K-K_{m}^{2}\right)\left(K-K_{n}^{2}\right)\left\{\left[J_{0}(K b)\right]^{2}+\left[Y_{0}(K b)\right]^{2}\right\}} d p,
$$

so that the result of changing the orders of integration and summation in Eq. 34 is

$$
\begin{aligned}
\sum_{m=0}^{\infty} N_{m}\left(\alpha_{m}^{2}+\beta_{m}^{2}\right) / p_{m}=-2 \int_{0}^{k} \frac{d p K^{2} a^{2}}{\left\{\left[J_{0}(K a)^{2}+Y_{0}(K b)\right]^{2}\right\}} \\
\cdot \sum_{0}^{\infty}\left\{\left(\frac{\beta_{m} \varphi_{m}(a)}{K^{2}-K_{m}^{2}}\right)^{2}+\sum_{0}^{\infty}\left(\frac{\alpha_{m} \varphi_{m}(a)}{K^{2}-K_{m}^{2}}\right)^{2}\right\} .
\end{aligned}
$$


The right-hand side of the equation is $\leq 0$. The left-hand side of this equation is, however, a non-negative quantity so that each side of the equation must be zero. It follows that

$$
\alpha_{m}, \beta_{m}=0 \text { for all } m \geq 0 .
$$

Given the infinite set of equations, it is necessary to know whether an approximate solution can be found by solving only the first $N+1$ equations for the unknowns $(n \leq N)$, taking all the remaining unknowns to be zero. We shall therefore prove the following

Theorem 2. (i) The determinant of the finite set of equations

$$
k N_{m}\left(2 \delta_{0 m}+a_{m} / i p_{m}\right)=\sum_{n=0}^{N} C_{n m} a_{n}, \quad 0 \leq m \leq N,
$$

does not vanish for any positive value of $\epsilon$

(ii) The unique solution of the finite number of equations is the set $\left\{a_{n}^{N}\right\}, 0 \leq n \leq N$, and $\lim \left(a_{n}-a_{n}^{N}\right)=0$ as $N \rightarrow \infty$.

The first part of the theorem is proved in the same way as in Theorem 1. For the second part we find from Eqs. 24 and 35 that

$$
k N_{m}\left(a_{m}-a_{m}^{N}\right) / p_{m}=\sum_{n=0}^{N} C_{n m}\left(a_{n}-a_{n}^{N}\right)+\sum_{n=N+1}^{\infty} C_{n m} a_{n} .
$$

Now from 19, using Parseval' Theorem, since $r^{1 / 2} L(r)$ is a function of integrable square in $a>r>b$, we see that

$$
\int_{b}^{a} r[L(r)]^{2} d r=\sum_{0}^{\infty}\left|a_{n}\right|^{2} N_{n}=0(1), \text { for } \epsilon>0 .
$$

Since $N_{n}=0\left(r^{-2}\right)$ as $n \rightarrow \infty$, a necessary condition for the convergence of this series is that as $n \rightarrow \infty$, for $\delta>0$,

$$
\left|a_{n}\right|=0\left(n^{1-\delta}\right) .
$$

It is shown in the Appendix that the infinite integral $C_{m n}$ is absolutely convergent for all $\epsilon>0$. As $n \rightarrow \infty\left|C_{m n}\right|=0\left(n^{-3}\right)$ so that

$$
\sigma_{m}^{N}=\left|\sum_{n=N+1}^{\infty} C_{m n} a_{n}\right|=0\left(N^{-1-\delta}\right)
$$

if $N$ is large enough, for all $m>0$.

Thus

$$
\left|\frac{k N_{m}\left(a_{m}-a_{m}^{N}\right)}{i p_{m}}-\sum_{n=0}^{N} C_{m n}\left(a_{n}-a_{n}^{N}\right)\right|=\sigma_{m}^{N},
$$

and since the determinant of the finite set of equations is non-zero, the solution of 36 must be of the form

$$
a_{m}-a_{m}^{N}=0\left(\sigma_{m}^{N}\right) .
$$

Therefore, we may approach as close as we like to a solution of the infinite set of equations if we take $N$ large enough, and from Theorem 1 this solution is a unique one. 


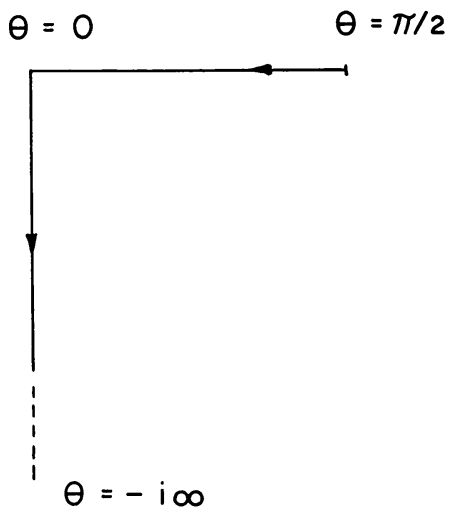

FIG. 3

Theorem 2 shows that we may obtain a useful approximation to the solution by solving for the first $N+1$ unknowns, and we are able to use a variational method to hasten the convergence to a correct result. This approach may be made in a more general problem in which there may be set up any number of propagating modes within the coaxial line.

\section{APPENDIX}

We transform the integrand in Eq. 23 by putting $p=k \cos \theta$. To correspond to the branch of $K$, we must take the path of integration in the complex $\theta$-plane as shown in the diagram (Fig. 3).

We are principally interested in the behavior of $C_{m n}$ for small values of the quantiy $\epsilon=k(a-b)$. We have to consider separately the possibility of the ratio $\rho=b / a$ approaching unity with $\sigma=k a$ not small, and also the case in which $\sigma$ is small and $\rho$ is not close to unity.

From the definition of the functions $\varphi_{n}(a)$ in Sec. III, it follows that as $\epsilon \rightarrow 0$

$$
\begin{aligned}
a \varphi_{n}(a) & =-2 \epsilon / n k \pi^{2}, & \text { for } & n \geq 1 \\
& =1, & & \text { for } n=0 .
\end{aligned}
$$

The functions $N_{n}$ defined in the same section behave in the following manner for small values of $\epsilon$. Thus, for any value of $\sigma$

$$
N_{0}=\ln \rho=\left\{\begin{array}{lll}
0(1) & \text { if } & \rho \leftrightarrow 1, \\
0(\epsilon) & \text { if } & \rho \rightarrow 1
\end{array}\right.
$$

and

$$
\begin{aligned}
N_{n} & =2\left\{1-\left[J_{0}\left(K_{n} a\right) / J_{0}\left(K_{n} b\right)\right]^{2}\right\} /\left(\pi K_{n}\right)^{2}, \\
N_{n} & =\frac{2 \epsilon^{2}}{\pi^{2} k^{2} n^{2}}\left\{1-\left[J_{0}(n \pi / 1-\rho) / J_{0}(n \pi \rho / 1-\rho)\right]^{2}\right\}, \\
& =0\left(\epsilon^{2}\right) \quad \text { if } \quad \rho \neq 1 \quad \sigma \rightarrow 0, \\
& =0\left(\epsilon^{3}\right) \text { if } \quad \rho \rightarrow 1 \quad \sigma \neq 0 .
\end{aligned}
$$


We shall first consider the convergence of the integral $C_{m n}$. On the path $C$ we have already established the bounded nature of the integrand near the origin, since we have examined its behavior in the $p$-plane near the branch point $p=+k$. This property is independent of the values of the parameters $\sigma$ and $\rho$. On the negative imaginary axis the integrand $I_{m n}$ is a real function involving modified Bessel functions of real argument.

We must examine the uniformity of convergence of the integral $\int_{0}^{\infty} I_{m n} d \varphi$ with respect to the parameters $\sigma$ and $\rho$. Let us take a small positive number $\sigma_{0}$ as close as we like to zero. Then, for $0<\rho \leq 1, \sigma \geq \sigma_{0}>0$, we may choose $\varphi_{0} \gg \sinh ^{-1}\left(1 / \sigma_{0}\right)$, and for $\varphi_{1}>\varphi_{0}$ we may use the asymptotic expansion of the modified Bessel functions. Since for all $n \geq 0, K_{n} \geq 0$, it follows that

$$
\left|\int_{\varphi_{1}}^{\infty} I_{m n} d \varphi\right| \leq \frac{2}{\pi} \int_{\varphi_{1}}^{\infty} \frac{d \varphi}{\sinh \varphi}\left[N_{n} \delta_{m n}+\frac{a \varphi_{n}(a) a \varphi_{m}(a)}{2 \sigma \sinh \varphi}\left(1+0(\sigma \sinh \varphi)^{-1}\right)\right] .
$$

Since for $\theta>\varphi_{1}, \sigma_{0 m h} \theta>1$, it follows from the convergence of the integral $\int_{\varphi_{1}}^{\infty} \operatorname{cosech} \theta d \theta$ that $C_{m n}$ is unformly convergent with respect to $\varphi$ and $\sigma$ in the given range. This is true for all $m, n \geq 0$.

Now consider $C_{00}$. The integrand vanishes for $\sigma=0, \rho \neq 1$ or $\sigma \neq 0, \rho=1$. It is clear that for $\rho \neq 1, \sigma$ is a factor of $C_{00}$, and for $\sigma=0,(1-\rho)$ is a factor. The derivative of the integrand with respect to $\rho$ is uniformly integrable in the same range of parameters, and this vanishes also for $\rho=1$; it follows that for $\sigma \neq 0, \rho 1, C_{00}=0\left(\epsilon^{2}\right)$ while for $\rho \neq 1, \sigma \rightarrow 0 C_{00}=0(\epsilon)$. The second derivative with respect to $\rho$ does not exist if $\rho=1$, $\sigma \neq 0$, nor does the first derivative with respect to $\sigma$ when $\sigma=0, \rho \neq 1$.

The magnitude of $C_{m n}$ for at least one of $m$ or $n$ not zero is determined by the magnitude of the integrand on the path $C$ at a finite distance from the origin. Since $K_{n} \sim n \pi k / \epsilon$, we find that

$$
\begin{array}{rlrl}
C_{0 n}=C_{n 0} & =0\left(\epsilon^{4}\right) \text { for } \rho \rightarrow 1, & & \sigma \neq 0, \\
& =0\left(\epsilon^{3}\right) \text { for } \quad \rho \neq 1, & \sigma \rightarrow 0,
\end{array}
$$

and that for both $m$ and $n$ not zero,

$$
\begin{array}{rlrl}
C_{m n} & =0\left(\epsilon^{5}\right) \text { for } \quad \rho \rightarrow 1, & & \sigma \neq 0, \\
& =0\left(\epsilon^{4}\right) \text { for } \quad \sigma \rightarrow 0, & \rho \neq 1 .
\end{array}
$$

\section{REFERENCES}

1. E. Jahnke, and F. Emde, Tables of higher functions, Leipzig, 1948

2. D. S. Jones, Quart. J. Mech. Appl. Math. 3, 420 (1950)

3. R. W. P. King, Theory of linear antennas, Harvard, 1956

4. N. Marcuvitz, Waveguide handbook, McGraw-Hill, New York, 1951

5. J. Meixner, Res. Rept., EM72, Inst. Math. Sci., New York Univ., 1954

6. G. N. Watson, Bessel functions, 2nd ed., Cambridge, 1944 